\title{
Escala de motivación situacional académica para estudiantes universitarios: desarrollo y análisis psicométricos
}

\author{
The academic motivation scale-situational for college students:
}

\section{Development and psychometric analysis}

\author{
Flavia Eugenia Bruno ${ }^{1}$, Mercedes Fernández Liporace ${ }^{2}$ y Juliana Beatriz Stover ${ }^{3}$ \\ ${ }^{1}$ Licenciada en Psicología. Doctoranda de la Universidad de Buenos Aires (UBA). Ayudante de \\ Trabajos Prácticos de Primera Categoría Regular en Teoría y Técnica de Exploración y Diagnós- \\ tico Psicológico, Módulo I, Cátedra II, Facultad de Psicología, UBA. \\ E-mail: flavia.bruno@yahoo.com.ar
}

${ }^{2}$ Doctora en Psicología. Investigadora Principal en el Consejo Nacional de Investigaciones Científicas y Técnicas (CONICET). Profesora Titular Regular en Teoría y Técnica de Exploración y Diagnóstico Psicológico, Módulo I, Cátedra II, Facultad de Psicología, Universidad de Buenos Aires.

${ }^{3}$ Doctora en Psicología. Especialista en Estadística para Ciencias de la Salud. Investigadora Asistente en el Consejo Nacional de Investigaciones Científicas y Técnicas (CONICET). Jefa de Trabajos Prácticos Regular en Teoría y Técnica de Exploración y Diagnóstico Psicológico,

Módulo I, Cátedra II, Facultad de Psicología, Universidad de Buenos Aires.

El presente trabajo fue subsidiado por la Universidad de Buenos Aires (Argentina) en el marco del Proyecto UBACyT 20020170100064B: "Variables Psicopatológicas y Psicoeducativas en Estudiantes Universitarios. Un Estudio Instrumental y Predictivo"

Instituto de Investigaciones en Psicología, Facultad de Psicología, Universidad de Buenos Aires. Ciudad Autónoma de Buenos Aires, Argentina

\section{Resumen}

La Teoría de la Autodeterminación (TAD) reconoce diferentes tipos de motivación, a lo largo de un continuo según el grado de autorregulación del comportamiento, que operan en tres niveles de generalidad: global, contextual y situacional. Este trabajo presenta el proceso de construcción y análisis psicométricos de la Escala de Motivación Situacional Académica desarrollada a partir de las hipótesis de la TAD y del Modelo Jerárquico de la Motivación Intrínseca y Extrínseca (MJMIE). Participaron del estudio 364 estudiantes $(62.6 \%$ mujeres,
$37.4 \%$ varones) de 18 a 35 años de edad ( $M$ $=22.97, D E=3.00)$ de diversas carreras de la Universidad de Buenos Aires (Argentina). Cinco jueces expertos analizaron evidencias de validez de contenido para los ítems y un grupo de alumnos aportó elementos para mejorar la validez aparente y la adecuación lingüística. Un análisis factorial exploratorio, a partir del método de Análisis Factorial de Rango Mínimo con rotación Oblimin directo, aisló una estructura trifactorial. La consistencia interna de las escalas se estimó mediante alfas ordinales, cuyos valores fueron en su totalidad adecuados. Las asociaciones 
significativas entre las escalas apoyaron la hipótesis teórica sobre el continuo propuesto en el modelo. Las correlaciones entre las escalas del instrumento con las de la Escala de Motivación Académica (Stover, de la Iglesia, Rial Boubeta y Fernández Liporace, 2012; Vallerand, Blais, Brierey Pelletier, 1989) fueron significativas al comparar dimensiones equivalentes y disímiles de ambas técnicas. Finalmente, se confeccionaron baremos para estudiantes universitarios locales. Los resultados, limitaciones, implicancias y líneas futuras de investigación se discuten a la luz del modelo de partida.

Palabras clave: Teoría de la Autodeterminación; Motivación situacional; Estudiantes universitarios; Validez; Consistencia interna.

\section{Abstract}

Self Determination Theory (Deci \& Ryan, 1985a) describes diverse types of motivation, situated throughout a theoretical continuum which comprises a decrease in the regulation of behaviors, from highest to lowest. These are intrinsic motivation, identified regulation, introjected regulation, external regulation, and amotivation. These types, their influential factors, mediators and outcomes, integrate a hierarchic model which entails three levels: global, contextual, and situational (Vallerand, 1997). This paper presents the design and analysis of psychometric features of the Academic Motivation Scale-Situational (AMS-S) aimed at the assessment of motivation towards specific academic activities under specific circumstances. It was developed on the grounds of the Self Determination Theory's hypotheses as well as the Hierarchic Model of Intrinsic and Extrinsic Motivation (HMIEM) posed by Vallerand (1997). The sample was composed of 364 colleges students (62.6\% females, $37.4 \%$ males) between 18 and 35 years old $(M=$ 22.97, $S D=3.00$ ) with different majors at the University of Buenos Aires (Argentine). Items were written up to represent each type of motivation formulated in the model, according to the question which must be borne in mind while responding to the scale: "Why are you at the faculty right now?" The original pool of items was evaluated by five experts in order to analyze content validity evidences. A further pilot study was performed with a small group of students, aiming at the exam of face validity evidences. The preliminary version of 44 items obtained up to then was analyzed on a standardization sample. Polychoric covariance matrices were employed once the univariate normality hypothesis was not verified for the items. The ordinal nature of data demanded such a treatment as well. The statistical package FACTOR (Lorenzo-Siva \& Ferrando, 2013) was used to develop an exploratory factor analysis with minimum rank factor analysis (MRFA) extraction method followed by direct Oblimin rotation $\left(\mathrm{KMO}=.887\right.$; Bartlett's Sphericity Test: $\chi^{2}=$ $4525.6(d f=351 ; p<.01)$. A 3-factor model which explained $68.01 \%$ of the common variance was isolated retaining 27 items (F1 explained $18.1 \%$, F2, $23.2 \%$, and F3, 26.8 $\%)$. Regarding the items content, the extracted factors were labeled as extrinsic motivation (F1), amotivation (F2), and intrinsic motivation (F3). Additionally, internal consistency of the scales was estimated by means of ordinal alphas, with adequate coefficients in all cases $(\mathrm{F} 1=.84, \mathrm{~F} 2=.95$, and F3 =.94 $)$. In order to test the self-determination continuum hypothesis entailed in the model, each dimension of the scale was correlated with the rest of them. Significant and positive associations $(p<.05)$ were found between the adjacent scales, and negative between the non-adjacent ones, thus endorsing theoretical statements. Correlations between the scales' scores and the similar ones of Academic Motivation Scale (Academic Motivation Scale-EMA in Spanish-Stover et al., 2012; Vallerand et al., 1989) were calculated to analyze convergent validity evidences. Significant though weak statistical associations $(p<.05)$ were found when comparing equivalent and oppo- 
site motivational types in both tests. Finally, statistical norms to be employed with local college population were calculated. Summarizing, up to the moment the scale has achieved adequate results in terms of preliminary validity evidences and reliability, supporting its appropriate psychometric quality to be used with local college students. The main weaknesses of the study rely in the sample size, which restricts wider generalizations, as well as the lack of semi-confirmatory factor analyses and psychometric studies on stability of measures, which must be developed in further steps. The analysis of the link between situational, contextual and global measures of motivation is also strongly suggested, taking into account the reciprocal effects among them.

Keywords: Self-determination Theory; Situational motivation; College students; Validity; Internal consistency.

\section{Introducción}

El estudio de la motivación ha despertado especial interés en el ámbito académico en virtud de su vinculación con los desempeños estudiantiles en diferentes estratos del sistema educativo. En cuanto al nivel universitario, se ha indicado que algunos perfiles motivacionales se hallaban con frecuencia presentes en alumnos con buen rendimiento académico (Boiché y Stephan, 2014; Gillet, Vallerand, Lafrenière y Bureau, 2013; Sturges, Maurer, Allen, Gatch y Shankar, 2016) mientras que otros eran característicos del estudiantado con mayores dificultades para cumplimentar los objetivos planteados por cada carrera; ello permitía incluso, predecir la probabilidad de deserción (e.g., Baars y Arnold, 2014). Así, contar con instrumentos para evaluar la motivación resulta crucial en pos de alcanzar una mayor comprensión del modo en que aquella impacta y modula las trayectorias académicas estudiantiles en el medio local. En este sentido, la Teoría de la Autodeterminación (en adelante TAD) aporta un robusto corpus teórico para el abordaje de esta temática, así como una prolífica producción de instrumentos destinados a su valoración.

\section{Teoría de la Autodeterminación}

La motivación alude a las distintas razones que guían la conducta humana. El constructo comprende tanto la energía como la dirección, persistencia y finalidad de los comportamientos, e incluye acciones e intenciones (Ryan y Deci, 2000). La TAD proponeuna conceptualización multidimensional que distingue diversas formas de motivación (Ver Figura 1) ubicadas a lo largo de un continuo dado por el grado de autonomía para la regulación de las conductas (Deci y Ryan, 1985a, 2004; Ryan y Deci, 2017).

En el polo de mayor autodeterminación se ubica la motivación intrínseca (MI), que permite llevar a cabo acciones por el placer y disfrute que derivan de su ejecución. Si bien originalmente el modelo asumió una perspectiva unidimensional en este punto, desarrollos posteriores provenientes de la investigación en contextos educativos diferenciaron tres subtipos: motivación orientada hacia el conocimiento, hacia el logro, y hacia las experiencias estimulantes (Vallerand et al., 1989). En el punto siguiente del continuo se posiciona la motivación extrínseca (en adelante ME); aquí las conductas constituyen un medio para alcanzar un fin y persiguen la obtención de recompensas o incentivos. Se reconocen cuatro subtipos que representan diversos niveles de internalización de los controles externos. Así, cuanto mayor es la internalización, mayor es la autodeterminación de los comportamientos (Ryan y Deci, 2000). La ME regulación integrada, primer subtipo, tiene lugar cuando la conducta es congruente con los valores y necesidades personales; se trata de la forma más autónoma de ME puesto que las regulaciones se asimilan completamente al sentido de sí mismo, y se supone se consolida hacia la edad adulta. La ME regulación identificada, por su parte, se manifiesta toda vez 
que el sujeto desarrolla actividades que considera su propia elección, aunque correspondan a motivos extrínsecos. Entre las formas menos autorreguladas de $\mathrm{ME}$ aparecen la ME regulación introyectada y la $\mathrm{ME}$ regulación externa. En la primera, las acciones se dirigen a evitar la culpa o enaltecer el ego; en la segunda, los comportamientos responden a exigencias externas y se efectúan a fin de evitar castigos $u$ obtener recompensas. En el extremo opuesto del continuo se sitúa la amotivación (A), que es unidimensional. A diferencia de las formas anteriores, aquí la conducta no se halla ni intrínseca ni extrínsecamente motivada; se trata de la ausencia de regulación de los comportamientos y por ende, la falta total de motivación. Aunque la acción volitiva es inexistente en la A, la mayoría de los autores la localizan en el punto más bajo del gradiente de autodeterminación (e.g., Howard, Gagné, Morin y Forest, 2018).

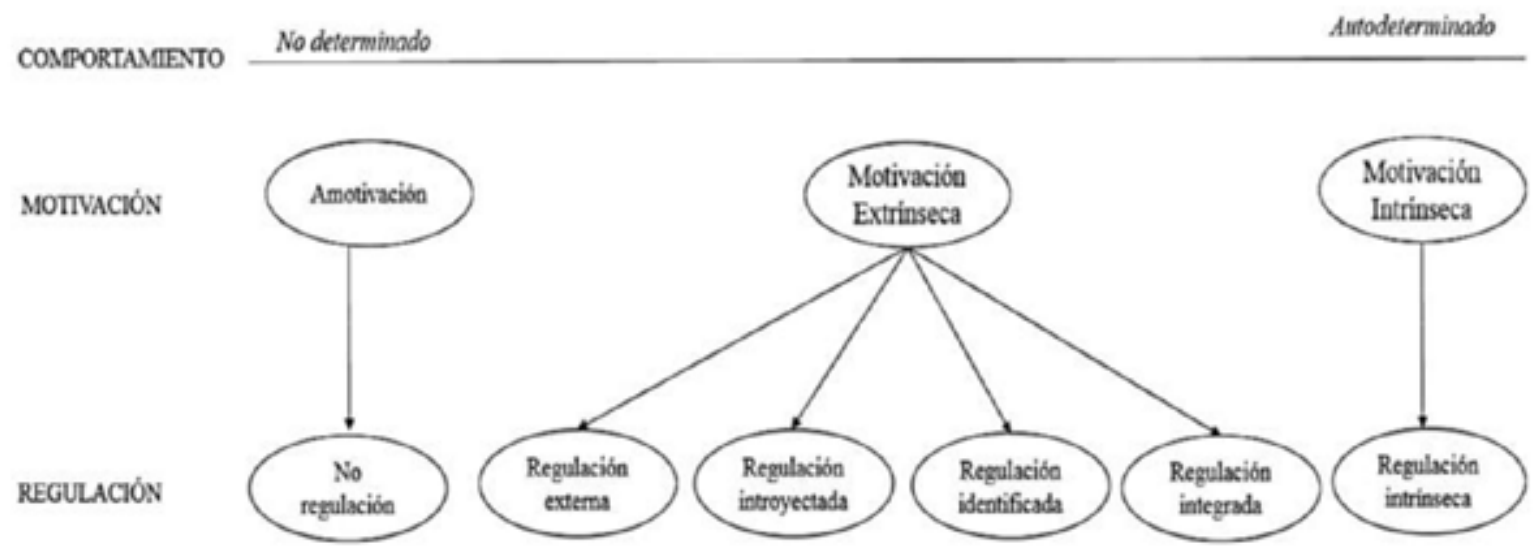

Figura 1. Continuo de Autodeterminación.

Ryan y Deci (2002) sostienen que este continuo no debe entenderse en términos de etapas que es necesario atravesaren el desarrollo vital para alcanzar una mayor autonomía. Por el contrario, un individuo puede adoptar diferentes tipos de regulación según los factores y demandas situacionales. Merece destacarse que este modelo continuo hace foco en el reconocimiento de formas autónomas $-\mathrm{MI}, \mathrm{ME}$ regulación integrada, $\mathrm{ME}$ regulación identificada $-\mathrm{y}$ controladas $-\mathrm{ME}$ regulación introyectada, $\mathrm{ME}$ regulación externa- $\mathrm{y}$ evita así la clásica distinción categorial entre variantes intrínsecas y extrínsecas de motivación (Ryan y Deci, 2017). Numerosas investigaciones han reportado asociaciones positivas entre los puntos adyacentes, y negativas entre los más distales del continuo, que han aportado sustento empírico para el modelo (e.g.,
Litalien, Morin, Gagné, Vallerand, Losier y Ryan, 2017). No obstante otros autores cuestionan estas hipótesis al proponer tipos motivacionales cualitativamente diferentes antes que formas articuladas en un gradiente de autonomía (Chemolli y Gagné, 2014).

A partir de los puntos principales de la TAD, Vallerand (1997) desarrolló el Modelo Jerárquico de la Motivación Intrínseca y Extrínseca (MJMIE) para describir el modo en que la motivación, sus determinantes, mediadores $\mathrm{y}$ consecuencias operan en tres niveles de generalidad: global, contextual y situacional. En un nivel global, más vinculado a la personalidad, el individuo desarrolla una orientación general y estable de interacción con el ambiente. Ello impacta en el modo habitual de interpretar los eventos y repercute en la iniciación y regulación de las conductas. La 
motivación contextual emerge en cada esfera específica de la actividad humana-educación, deporte, ocio, trabajo, entre otras- en las que los factores inherentes a cada dominio ejercen gran influencia. El nivel situacional, en cambio, analiza la motivación en momentos temporales concretos; por ejemplo, en una actividad realizada en una asignatura en particular en un año, mes y día determinados. Se trata del nivel más inestable ya que depende de la variabilidad y las características de cada actividad particular.

En cada uno de los niveles descriptos los factores sociales intervienen en la motivación a través de las necesidades psicológicas básicas de competencia, autonomía y vinculación. En función de su satisfacción emergen los distintos tipos de motivación MI, ME, A-. Asimismo, la motivación en un nivel influye sobre la del nivel inferior más próximo, y viceversa (Lavigne y Vallerand, 2010). Esta dinámica recursiva ha sido examinada en diversos ámbitos como el deportivo y el académico (Blanchard, Mask, Vallerand, de la Sablonnière y Provencher, 2007; Guay, Mangeau y Vallerand, 2003; Nuñez y León, 2018).

\section{Instrumentos de evaluación}

Actualmente existen múltiples instrumentos para valorar aspectos motivacionales en cada uno de los tres niveles de generalidad. No obstante al focalizar en el área educativa algunos adquieren mayor relevancia en virtud de sus propiedades psicométricas establecidas y de la constante investigación aplicada derivada de su utilización sostenida. Para el nivel global se destacan la Escala de Orientaciones Generales de Causalidad (Deci y Ryan, 1985b), que ha sido adaptada a población local (Brenlla, Messina y Aranguren, 2013) y la Escala de Motivación Global (Guay, Blais, Vallerand y Pelletier, 1999), que no cuenta con una versión adaptada a nuestro medio. La Escala de Motivación Académica (EMA, Vallerand et al., 1989), diseñada para el nivel contextual en el dominio educativo, cuenta con adaptaciones realizadas en Argentina destinadas a estudiantes de nivel medio y universitario (Aisenson, Valenzuela, Celeiro, Bailac y Legaspi, 2010; López, 2008; Stover et al., 2012). Sin embargo, para el nivel situacional se dispone de un número reducido de escalas cuya característica principal es la versatilidad en su aplicación, que las hace generalizables a cualquier dominio y situación. En este último grupo, la Escala de Motivación Situacional (Guay, Vallerand y Blanchard, 2000), de origen franco-canadiense, se ubica como el principal instrumento debido a su amplia difusión y a la gran cantidad de estudios psicométricos con los que cuenta. Se trata de una medida de autorreporte compuesta por 16 ítems y formato de respuesta Likert de 7 posiciones $(1=$ No se corresponde en absoluto, $7=\mathrm{Se}$ corresponde exactamente), que interroga sobre los motivos por los que el sujeto se encuentra comprometido en una actividad concreta. Evalúa cuatro de los ocho subtipos de motivación teorizados en la TAD: MI, ME regulación identificada, $\mathrm{ME}$ regulación externa y $\mathrm{A}$. $\mathrm{Al}$ respecto, los autores argumentaron que su decisión de evaluar la MI como constructo unidimensional, así como la exclusión de las escalas correspondientes a ME regulación integrada e introyectada del modelo, respondió exclusivamente a la necesidad de contar con un instrumento breve, versátil y de fácil aplicación (Guay et al., 2000). La escala posee estudios de evidencia de validez de constructo (estimada mediante análisis factoriales exploratorios y confirmatorios). En cuanto a la dimensionalidad, la solución de cuatro factores obtenida en factorizaciones exploratorias explicó el $65 \%$ de la varianza, porcentual más que satisfactorio para escalas de autoadministración. En estudios factoriales confirmatorios se han reportado diversos índices que dan cuenta de la bondad de ajuste del modelo propuesto. También cuenta con evidencia de validez concurrente con medidas de motivación contextual correspondientes a las áreas académica y deportiva, según el caso (Guay et 
al., 2000; Standage, Treasure, Duda y Prusak, 2003). En relación con la consistencia interna, sus escalas presentaron valores adecuados con alfas de Cronbach que varían entre .77 y .95. Al examinar su estabilidad temporal mediante el procedimiento de test-retest se hallaron valores aceptables, aunque los autores indican que variaciones en las puntuaciones deberían ser esperables por tratarse de una medida del estado actual (Guay et al., 2000). Análisis posteriores aportaron nuevas evidencias en favor de su adecuada calidad psicométrica (Lonsdale, Sabiston, Taylor y Ntoumanis, 2011), así como algunas variaciones respecto de su dimensionalidad, que no alteraron mayormente la solución tetrafactorial. Esto llevó a la conformación de versiones algo más breves como la estadounidense y la portuguesa, de 14 ítems (Gamboa, Valadas y Paixão, 2017; Standage et al., 2003). Una mención especial merece la adaptación efectuada para población española, que es la única en habla hispana (Martin-Albo, Núñez y Gavarro, 2009). Los estudios de validez de constructo de esta última incluyeron únicamente análisis factoriales confirmatorios. Al igual que en otros trabajos, los índices de ajuste del modelo de 16 ítems no resultaron satisfactorios, por lo que se ensayaron diversas reespecificaciones hasta alcanzar una estructura de 14 ítems con mayor bondad de ajuste, e invarianza factorial según sexo. Cuenta con evidencias de validez concurrente con la Escala de Motivación Educativa (Núñez, Martín-Albo y Navarro, 2005). El testeo de la hipótesis del continuo de autodeterminación es consistente con lo informado en la literatura previa (e.g., Guay et al., 2000). Para el estudio de la confiabilidad se exploró la consistencia interna de las cuatro escalas y se obtuvieron coeficientes alfa de Cronbach entre .81 y .85 , más elevados que los reportados para el modelo original de 16 ítems.

En nuestro país existe un área de vacancia en cuanto a la medición de la motivación situacional, por lo que resulta necesario adaptar y/o construir instrumentos psicométricos para tal fin. La escala antes descripta (Guay et al., 2000) surge como primera opción. Sin embargo, su versatilidad en cuanto a su aplicabilidad en diversas situaciones se vuelve aquí, paradójicamente, una desventaja. Si se tiene en cuenta que en cada dominio vital concreto los motivos por los que las personas se involucran en tareas puntuales varían y dependen de cada situación, contar con instrumentos que capturen tal especificidad se vuelve fundamental, de cara a su uso en contextos bien delimitados. En atención a lo anterior, el presente trabajo se propone el desarrollo de una escala de motivación situacional aplicable a las contingencias de la vida académica universitaria. Adicionalmente, se busca analizar sus propiedades psicométricas - evidencias de validez de contenido, de facies, de constructo y concurrente, más confiabilidad en términos de consistencia interna- así como poner a prueba la hipótesis del continuo de autodeterminación planteada por el modelo y construir baremos para su utilización en población universitaria local.

\section{Método}

\section{Diseño}

Se trabajó con un diseño no experimental, transversal, de tipo psicométrico y correlacional.

\section{Participantes}

El tipo de muestreo utilizado fue no probabilístico. Participaron 364 estudiantes de la Universidad de Buenos Aires (62.6\% mujeres, $37.4 \%$ varones) entre 18 y 35 años $(M=$ $22.97, D E=3.00)$. Para mejorar la representatividad poblacional se trabajó con alumnos que cursaban sus estudios en las Facultades de Arquitectura, Diseño y Urbanismo, Farmacia y Bioquímica, Derecho, Filosofía y Letras, Psicología, Ciencias Exactas y Naturales y Ciencias Económicas, y se respetaron las distribuciones según edad y sexo reportadas para cada unidad 
académica en estadísticas de organismos oficiales (Ministerio de Educación, 2013). En cuanto a los criterios de exclusión fueron eliminados del estudio quienes se encontraban por fuera del rango de edad establecido, que corresponde al de la mayoría de los cursantes de la Universidad, así como aquellos que omitieron responder más del $5 \%$ de alguno de los tests.

\section{Instrumentos}

\section{Encuesta de datos sociodemográficos y académicos}

A fin de caracterizar la muestra, se recabaron datos sobre aspectos sociodemográficos tales como edad y sexo, por un lado, y académicos, como Facultad y carrera, por otro.

\section{Escala de Motivación Académica (EMA, Stover at al., 2012; Vallerand et al. 1989)}

Esta escala se utilizó como criterio externo. Explora los motivos por los que los estudiantes asisten a la Facultad. Sus subescalas son MI hacia las experiencias estimulantes, MI hacia el logro, MI hacia el conocimiento, ME regulación identificada, ME regulación introyectada, ME regulación externa y $\mathrm{A}$. La ME regulación integrada fue descartada del modelo por tratarse de un subtipo que aparece más tardíamente, en la adultez (Stover et al., 2012). Está conformada por 27 ítems con respuesta Likert de cuatro posiciones (Totalmente en desacuerdo, Un poco de acuerdo, Bastante de acuerdo, Totalmente de acuerdo). La adaptación argentina (Stover at al., 2012) cuenta con estudios de validez de contenido, de facies, y de constructo -mediante análisis factorial confirmatorio- más análisis de la consistencia interna de sus escalas con alfas ordinales que oscilan entre .59 y .82 .

\section{Escala de Motivación Situacional Académica}

Para la construcción de la escala, objeto de este trabajo, se diseñaron reactivos que consideraron siete dimensiones del constructo -MI hacia las experiencias estimulantes, MI hacia el logro, MI hacia el conocimiento, $\mathrm{ME}$ regulación introyectada, $\mathrm{ME}$ regulación identificada, ME regulación externa, A- en atención a las hipótesis teóricas de la TAD y la adaptación local de la EMA (Stover et al., 2012; Vallerand et al., 1989) que excluyen la ME regulación integrada. Adicionalmente se tomaron algunos de los ítems de aquel test, que valora la vertiente contextual del constructo, y se efectuaron alteraciones en su contenido para volverlos congruentes con la consigna general del nuevo instrumento -¿Por qué estás en este momento en la Facultad?-. Por otra parte, también se recurrió a un pequeño grupo de alumnos $(\mathrm{n}=38)$ a los que se les solicitó que informaran por escrito los motivos por los que se encontraban en ese momento en la Facultad. Esto último tuvo por finalidad sumar un criterio empírico para la confección de los ítems. Como resultado de todo este proceso se obtuvo una primera versión de la escala constituida por 44 afirmaciones y un formato de respuesta tipo Likert con cinco opciones de respuesta mediante las que el estudiante evaluado indicaba su grado de acuerdo con los reactivos presentados (Totalmente en desacuerdo, Algo en desacuerdo, $\mathrm{Ni}$ de acuerdo ni en desacuerdo, Algo de acuerdo, Totalmente de acuerdo).

\section{Procedimiento}

En primer lugar se convocó a un grupo de cinco expertos en psicometría con amplia experiencia en el estudio de variables psicológicas asociadas a diversos fenómenos educativos. La tarea encomendada requería la valoración independiente de cada uno de los reactivos de la escala con el objetivo de investigar su capacidad para representar la dimensión para la que habían sido creados, así como la adecuada representación de cada dimensión teórica en forma proporcionada (estudio de validez de contenido). Luego se seleccionó una muestra de 28 alumnos a quienes 
que se les administró esta versión preliminar y se les pidió que juzgaran la comprensión y pertinencia de la consigna en general y de los reactivos y formato de respuesta en particular (estudio de validez de facies y adecuación lingüística). La versión refinada del instrumento fue administrada a la muestra de tipificación definitiva descripta anteriormente. Previamente se contactó a docentes de distintas Facultades de la Universidad de Buenos Aires y se acordaron días y horarios de clases para la recolección colectiva de datos en esas instancias. Las administraciones estuvieron bajo la supervisión de una psicóloga entrenada, quien informó al alumnado sobre la finalidad de la investigación y las pautas de completamiento de los protocolos. Los estudiantes participaron de forma voluntaria, sin retribución económica, y prestaron su conformidad mediante un consentimiento informado. En él se les notificaban los objetivos generales del estudio, la posibilidad de desistir de responder o interrumpir la respuesta en cualquier momento, y se les garantizaban el anonimato y la confidencialidad de los datos y resultados. El estudio contó con los avales institucionales correspondientes.

\section{Análisis de datos}

Para la estimación del acuerdo interjueces se calcularon coeficientes Aiken para cada uno de los ítems valorados. Para el análisis de la dimensionalidad del instrumento se utilizó el método de extracción denominado análisis factorial de rango mínimo y rotación oblicua (Oblimin directo), en sintonía con las directrices actuales para la realización de este tipo de estudios (e.g., Lloret-Segura, Ferreres-Traver, Hernández-Baeza y TomásMarco, 2014). A su vez se empleó como input la matriz de covarianza policórica debido a que los reactivos se responden mediante una escala ordinal (Muthén y Kaplan, 1985). Se calcularon coeficientes alfa ordinales para el análisis de la consistencia interna de las escalas obtenidas en el paso anterior.
Ambos procedimientos -dimensionalidad y consistencia interna- fueron realizados con el programa estadístico FACTOR (Lorenzo-Seva y Ferrando, 2013). Luego se efectuaron correlaciones entre las dimensiones de la Escala de Motivación Situacional Académica con el objetivo de poner a prueba la hipótesis del continuo de autodeterminación planteada teóricamente. El mismo procedimiento fue empleado para aportar evidencias de validez concurrente al examinar el grado, signo y significación de asociación entre las escalas del instrumento diseñado y las dimensiones de la Escala de Motivación Académica. Finalmente se calcularon percentiles correspondientes a las puntuaciones para los factores del instrumento a fin de confeccionar normas estadísticas para población universitaria. Todos estos análisis fueron realizados con PASW Statistics for Windows, Versión 18.0 (SPPS INC, 2009).

\section{Resultados}

\section{Validez de contenido y de facies}

A fin de aportar evidencias de validez de contenido se convocó a cinco jueces expertos para juzgar la pertinencia y adecuación de cada uno de los reactivos de la escala. Una vez obtenidas todas las evaluaciones se estimó el grado de acuerdo interjueces mediante el cálculo de los coeficientes Aiken para cada ítem. Se fijó como mínimo aceptable el valor .60 , equivalente al acuerdo de al menos tres de los cinco jueces. Como resultado, se reubicaron tres elementos en dimensiones distintas a la original para la que habían sido diseñados debido a que se hallaron acuerdos iguales o superiores a .60 en aquella nueva dirección.

Para finalizar la etapa de diseño y ajuste del banco inicial de ítems, se llevó a cabo una administración piloto con un grupo pequeño de estudiantes, con el objeto de obtener evidencias de validez aparente y de adecuar lingüísticamente consigna, ítems y escala de respuestas. La instrucción impartida incluía, 
además de responder al test, la realización de críticas y comentarios referidos a la redacción de la consigna general y de los ítems, así como al formato de respuesta. Los resultados del piloto permitieron introducir cambios menores en la formulación de algunos ítems de la escala, sin alterar su sentido(Por ejemplo, el ítem 32, Porque sé que mi formación universitaria es costeada directa o indirectamente por otras personas, y no puedo darme el lujo de faltar el día de hoy se modificó por Porque mis estudios son costeados por otros (Estado, mis padres, etc.), y no puedo darme el lujo de faltar el día de hoy).

\section{Análisis descriptivo}

Previo a los estudios factoriales se analizó la normalidad univariante de los ítems. Se calcularon las medias y desviaciones típicas para cada uno, así como la asimetría y curtosis, encontrándose valores absolutos elevados (superiores a2) para todos los reactivos de amotivación. Dado el incumplimiento de la normalidad univariante y la característica ordinal de los datos se decidió su posterior tratamiento para los análisis factoriales a partir de matrices de covarianza policóricas (Muthén y Kaplan, 1985).

\section{Validez de constructo}

Para el estudio de la dimensionalidad se utilizó el método de análisis factorial de rango mínimo el cual permite obtener el porcentaje de varianza común explicada por cada factor aislado, más rotación oblicua Oblimin directo. Inicialmente se forzó a siete factores para seguir los lineamientos teóricos de partida.
La solución factorial obtenida presentaba ciertas inconsistencias puesto que, a excepción de cuatro factores, el resto reunía reactivos de muy diversa índole y se dificultaba demasiado su interpretación desde un punto de vista conceptual. Asimismo, el método de Análisis Paralelo (Horn, 1965) sugería soluciones de cuatro y tres factores según se considerase la media o el percentil 95, respectivamente. Por tal motivo se decidió aislar, en primer término, un modelo de cuatro factores en consonancia con la estructura referida en otras escalas que evalúan la motivación situacional (e.g., Guay et al., 2000). Tras sucesivos ensayos en los que se suprimieron reactivos que exhibían cargas factoriales inferiores a .40 o saturaciones simultáneas mayores o iguales a ese valor en más de una dimensión, se arribó a una solución trifactorial con mayor sentido teórico que la tetrafactorial.

La adecuación muestral para el número de variables analizadas resultó satisfactoria, por lo que quedó debidamente justificada la realización de los cálculos antes detallados (KMO $=.887$; Prueba de Esfericidad de Bartlett: $\chi^{2}$ $=4525.6, d f=351 ; p<.01)$. El modelo definitivo retuvo 27 de los 44 reactivos inicialmente diseñados, agrupados en tres factores que explican el $68.01 \%$ de la varianza común $(\mathrm{F} 1=18.1 \%$; F2 = $23.2 \%$; F3 = $26.8 \%)$. En función del contenido de los ítems los factores se denominaron ME (Factor 1), A (Factor 2) y MI (Factor 3). Cabe destacar que mientras ME reúne todas las regulaciones extrínsecas teorizadas, MI sólo cuenta con ítems que inicialmente representaban la MI hacia el logro y la MI hacia el conocimiento. La Tabla 1 resume los resultados de la solución obtenida. 
Tabla 1

Escala de Motivación Situacional Académica. Análisis Factorial Exploratorio.

\begin{tabular}{|c|c|c|c|c|}
\hline & Ítem & $\begin{array}{l}\text { ME } \\
\text { (F1) }\end{array}$ & $A(\mathbf{F} 2)$ & $\begin{array}{r}\text { MI } \\
\text { (F3) }\end{array}$ \\
\hline 4 & Porque en mi opinión, es importante asistir hoy a clases. & .56 & -.36 & 09 \\
\hline 5 & $\begin{array}{l}\text { Me sentiría mal conmigo mismo/a si no cumplo con mis deberes de } \\
\text { estudiante el día de hoy. }\end{array}$ & .58 & -.17 & .00 \\
\hline 6 & Para no acumular inasistencias & .71 & .14 & -.17 \\
\hline 11 & $\begin{array}{l}\text { Porque sí elegí estudiar una carrera, es importante asistir a la mayor } \\
\text { cantidad de clases que pueda. }\end{array}$ & .58 & -.33 & .02 \\
\hline 18 & $\begin{array}{l}\text { Porque mi futuro profesional depende de aprovechar cada día de } \\
\text { cursada, incluyendo el de hoy. }\end{array}$ & .50 & -.17 & .25 \\
\hline 19 & Siento que es un compromiso venir hoy. & .60 & -.04 & .17 \\
\hline 20 & Para evitar quedarme libre por faltas. & .58 & .31 & -.11 \\
\hline 25 & $\begin{array}{l}\text { Porque forma parte de mi rutina como estudiante universitario/a estar } \\
\text { hoy aquí. }\end{array}$ & .65 & -.05 & -.03 \\
\hline 30 & $\begin{array}{l}\text { Porque asistiendo a clases podré mejorar mi rendimiento en la } \\
\text { materia. }\end{array}$ & .49 & -.18 & .29 \\
\hline 32 & $\begin{array}{l}\text { Porque mis estudios son costeados por otros (Estado, mis padres, } \\
\text { etc.), y no puedo darme el lujo de faltar el día de hoy. }\end{array}$ & .49 & .19 & .08 \\
\hline 34 & $\begin{array}{l}\text { Para no malgastar una falta que seguramente necesitaré más adelante } \\
\text { en el cuatrimestre. }\end{array}$ & .49 & .35 & .08 \\
\hline 37 & $\begin{array}{l}\text { Porque creo que soy un/a mejor estudiante si asisto a todas las clases } \\
\text { de las asignaturas que curso hoy. }\end{array}$ & .63 & .01 & .22 \\
\hline 7 & $\begin{array}{c}\text { Ahora no lo sé, siento que hoy estoy perdiendo el tiempo en la } \\
\text { facultad. }\end{array}$ & .10 & .72 & -.17 \\
\hline 14 & No sé para qué estoy hoy acá; no le encuentro sentido. & -.01 & .85 & .00 \\
\hline 21 & No entiendo para qué vine hoy, podría estar haciendo otra cosa. & .03 & .90 & .01 \\
\hline 28 & No lo sé; no puedo entender qué hago hoy acá en la facultad. & .02 & .94 & .04 \\
\hline 35 & $\begin{array}{l}\text { No sé por qué estoy en este momento en la facultad, sin duda, tengo } \\
\text { cosas más importantes que hacer. }\end{array}$ & -.02 & .89 & -.00 \\
\hline 39 & $\begin{array}{l}\text { No sé por qué estoy en este momento en la facultad; sin duda, tengo } \\
\text { cosas más interesantes que hacer. }\end{array}$ & -.08 & .91 & -.00 \\
\hline 2 & $\begin{array}{l}\text { Porque me supero a mí mismo/a cuando logro entender los temas de } \\
\text { la clase de hoy. }\end{array}$ & .06 & .05 & .82 \\
\hline 3 & Porque disfruto aprendiendo los temas nuevos que veremos hoy. & -.13 & -.05 & .82 \\
\hline 9 & $\begin{array}{c}\text { Por el placer que experimento cuando observo que hoy mejoré mis } \\
\text { destrezas/habilidades/conocimientos respecto de algunos de los } \\
\text { contenidos de la/s asignatura/s que curso hoy. }\end{array}$ & .07 & .08 & .84 \\
\hline 10 & $\begin{array}{l}\text { Porque me gusta aprender más sobre algunos temas de la/s clase/s de } \\
\text { hoy. }\end{array}$ & -.05 & -.08 & .78 \\
\hline 17 & $\begin{array}{c}\text { Porque me gusta profundizar mis conocimientos sobre algunos } \\
\text { contenidos de la/s clase/s de hoy. }\end{array}$ & -.05 & -.03 & .84 \\
\hline
\end{tabular}




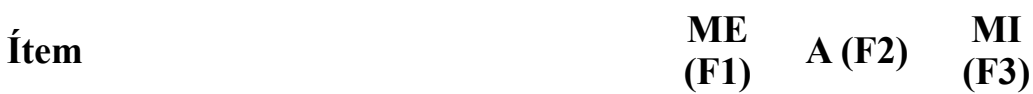

\begin{tabular}{ccccc}
\hline 23 & $\begin{array}{c}\text { Por el placer que experimento cuando me doy cuenta que hoy me } \\
\text { superé a mí mismo/a al poder realizar actividades que me resultaban } \\
\text { complejas. }\end{array}$ & .05 & .01 & .83 \\
\hline 24 & $\begin{array}{c}\text { Porque disfruto de comprender mejor algunos temas de la/s clase/s de } \\
\text { hoy. }\end{array}$ & .04 & -.02 & .84 \\
\hline 36 & $\begin{array}{c}\text { Porque me gusta relacionar conocimientos previos con el contenido } \\
\text { de la clase de hoy. }\end{array}$ & -.06 & .10 & .79 \\
\hline 43 & $\begin{array}{c}\text { Porque disfruto de venir a clase para comprender mejor los } \\
\text { conceptos y bibliografía en general. }\end{array}$ & .12 & -.23 & .57 \\
\hline
\end{tabular}

Notas: ME: Motivación extrínseca; A: Amotivación; MI: Motivación intrínseca.

\section{Validez concurrente}

Con el objeto de aportar evidencias de validez empírica concurrente se correlacionaron las dimensiones de la Escala de Motivación Situacional Académica con las de la Escala de Motivación Académica (EMA, Stover et al., 2012). Se hallaron asociaciones significativas positivas y mayoritariamente moderadas, entre las escalas de motivación situacional y contextual que valoran dimensiones equivalentes (por ejemplo, entre MI situacional y las tres escalas de MI de la
EMA); se observaron correlaciones negativas y débiles al considerar dimensiones que representan niveles de autodeterminación situados en los polos opuestos del gradiente (entre A situacional y los diversos subtipos de MI contextual). Por su parte, las escalas de ME contextual de mayor autodeterminación exhibieron correlaciones positivas, aunque débiles con MI situacional (por ej., entre MI situacional y ME identificada contextual). En la Tabla 2 se presentan los coeficientes de correlación obtenidos en esta sección.

Tabla 2

Correlaciones entre las escalas de la EMA y las escalas de la Escala de Motivación Situacional Académica (EMSA).

\begin{tabular}{cccccccc}
\multirow{2}{*}{ EMSA } & MI ee & MI log & MI con & ME Idfc & ME Int & ME Ext & A \\
\cline { 2 - 8 } & $.50^{* *}$ & $.52^{* *}$ & $.53^{* *}$ & $.16^{* *}$ & - & - & $-.23^{* *}$ \\
MI & - & - & $.24^{* *}$ & $.45^{* *}$ & $.47^{* *}$ & $.40^{* *}$ & - \\
ME & $-.21^{* *}$ & $-.27^{* *}$ & $-.19^{* *}$ & $-.11^{*}$ & - & - & $.34^{*}$
\end{tabular}

Notas: ${ }^{* *} \mathrm{p}<.01$ bilateral; ${ }^{*} \mathrm{p}<.05$. MI: Motivación intrínseca; MI ee: Motivación intrínseca hacia las experiencias estimulantes; MI log: Motivación intrínseca hacia el logro; MI con: Motivación intrínseca hacia el conocimiento; ME: Motivación extrínseca; ME Idfc: Motivación extrínseca regulación identificada; ME Int: Motivación extrínseca regulación introyectada; ME Ext: Motivación extrínseca regulación externa; A: Amotivación

\section{Confiabilidad - Consistencia interna}

Dado el carácter ordinal de los datos se calcularon coeficientes alfa ordinales para estimar la consistencia interna de las tres escalas obtenidas en el análisis exploratorio de 
los ítems (Elosúa y Zumbo, 2008). Los índices obtenidos resultaron adecuados (Factor1: .84; Factor 2: .95; Factor 3: . 94).

\section{Continuo de autodeterminación}

Se calcularon correlaciones entre las escalas del instrumento a fin de someter a prueba la hipótesis del continuo de autodeterminación que sostiene la TAD. Se observaron asociaciones significativas, aunque débiles, entre MI y $\mathrm{ME}(r=.16 ; p<.05)$, y entre MI y A $(r=-.31$; $p<.05)$, tratándose en el primer caso de puntos adyacentes, y de distales en el segundo.

\section{Normas regionales}

En la Tabla 3 se presenta el baremo correspondiente a estudiantes universitarios residentes en Ciudad Autónoma de Buenos Aires y alrededores para su uso en el ámbito de aplicación.

Tabla 3

Baremo de la Escala de Motivación Situacional Académica.

\begin{tabular}{cccc} 
Percentil & MI & ME & A \\
\hline 5 & 19 & 26 & 6 \\
10 & 24 & 29 & 6 \\
15 & 27 & 32 & 6 \\
20 & 29 & 34 & 6 \\
25 & 31 & 35 & 6 \\
30 & 32 & 37 & 6 \\
35 & 33 & 38 & 6 \\
40 & 34 & 39 & 6 \\
45 & 35 & 40 & 6 \\
50 & 35 & 42 & 6 \\
55 & 36 & 42 & 6 \\
60 & 37 & 44 & 7 \\
65 & 37 & 44 & 7 \\
70 & 38 & 46 & 8 \\
75 & 40 & 47 & 9 \\
80 & 41 & 48 & 10 \\
85 & 42 & 50 & 12 \\
90 & 44 & 53 & 14 \\
95 & 45 & 55 & 17
\end{tabular}

\section{Discusión}

En las últimas décadas el estudio de la motivación ha dado lugar a una gran producción de investigaciones en el terreno educativo dado que este constructo parece capaz de aportar una mayor comprensión sobre la adquisición de diversos aprendizajes, el logro o frustración de los objetivos académicos, así como también dar cuenta de la permanencia estudiantil en los distintos estratos del sistema educativo (Baars y Arnold, 2014; Boiché y Stephan, 2014; Gillet et al., 2013. En consecuencia, contar con instrumentos de 
evaluación válidos y confiables que permitan abordar esta temática reviste vital importancia. Existen diversas medidas para evaluar la motivación desde los puntos de vista global, contextual y situacional basados en la TAD (Deci y Ryan, 1985a, 2000) y en el MJMIE propuesto por Vallerand (1997). Sin embargo, aunque se dispone de escalas para examinar los dos primeros niveles, escasean los instrumentos que valoren la motivación situacional. En virtud de ello, el presente trabajo tuvo por finalidad el desarrollo de una escala para medir la motivación situacional académica en estudiantes universitarios.

Tras la confección del banco inicial de ítems se realizaron diversos análisis psicométricos que permitieron obtener evidencias preliminares acerca de la validez - de contenido, aparente, de constructo y concurrente- y la consistencia interna de la Escala de Motivación Situacional Académica aquí presentada. Si bien los estudios iniciales -juicio experto $\mathrm{y}$ adecuación lingüística en administraciones piloto- arrojaron resultados satisfactorios, restan aún algunas reflexiones respecto de los demás resultados a fin de elucidar los posibles alcances de la escala y las futuras líneas de trabajo.

En cuanto a la validez de constructo, la solución de tres factores resulta diferente respecto de la obtenida para la Escala de Motivación Situacional (Guay et al., 2000). Esta última distingue entre formas más $\mathrm{y}$ menos autorreguladas de $\mathrm{ME}$, mientras que en el instrumento aquí presentado todos los tipos de motivación - MI, ME, A- están representados unidimensionalmente. Al respecto, se le puede objetar su capacidad para capturar de manera fehaciente la gradualidad de autonomía postulada por la TAD, siendo esta una de sus principales limitaciones. Por otra parte, la nueva escala diseñada posee la ventaja de ser exclusiva para contextos académicos puesto que sus ítems refieren a motivos vinculados con las actividades áulicas, en contraste con los reactivos de la Escala de Motivación Situacional, cuya ambigüedad los vuelve adaptables a cualquier contexto tal como lo han probado las investigaciones aplicadas (Guay et al., 2000; Standage et al., 2003).

Los estudios de validez concurrente obtuvieron asociaciones positivas, entre débiles y moderadas, entre las escalas motivacionales contextuales y situacionales que referían a un mismo aspecto del constructo, y negativas entre las que representaban dimensiones cualitativamente diferentes, tal como se informó en investigaciones anteriores (e.g. Martín-Albo et al., 2009) y en consonancia con el modelo teórico. En cuanto a la fuerza de la asociación, la debilidad reportada en algunos casos resulta lógica desde un punto de vista conceptual si se atiende a la naturaleza inestable que caracteriza la motivación situacional. Por ejemplo, un alumno puede exhibir un perfil motivacional autodeterminado hacia las actividades académicas -motivación contextual-pero no hallarse intrínsecamente motivado hacia una tarea puntual en la que está involucrado en un momento dado -motivación situacional-.

Con respecto al estudio de la confiabilidad, los coeficientes alfa ordinales resultaron adecuados para todos los factores, dando cuenta de una buena consistencia interna.

En cuanto a la hipótesis del continuo, la asociación positiva entre formas que implican tipos motivacionales disímiles-MI y MEresulta lógica si se comprende que en ambos casos la conducta se halla determinada de algún modo. Por el contrario, la relación inversa entre A y MI responde a la ausencia de regulación que caracteriza a la primera respecto de la mayor autonomía que la segunda encarna. Los hallazgos siguen la misma línea que 1o informado por la literatura previa a propósito de los vínculos entre subtipos próximos y distales (Guay et al., 2000; Litalien et al., 2017; Lonsdale et al., 2011).

De lo analizado hasta aquí se desprenden algunas limitaciones del estudio que es necesario mencionar. La primera alude al tamaño y características muestrales, que restringen la posibilidad de generalizar los resultados obtenidos. Si bien se procuró incluir estudiantes 
de diversas carreras, no se obtuvo la participación de un número considerable de alumnos de cada una. A la vez, al trabajarse con un muestreo intencional, algunas disciplinas no han sido incluidas. En segundo lugar, al tratarse del desarrollo de una nueva medición, solamente se estudió la validez factorial de modo exploratorio, por lo que nuevos trabajos deben analizar sucesivamente la dimensionalidad, preferentemente a través de análisis factoriales semi-confirmatorios que impongan diversos grados de restricción al modelo (Lloret-Segura et al., 2014).

En cuanto a las futuras líneas de investigación, resulta deseable el aporte de nuevas evidencias acerca de las propiedades psicométricas del instrumento presentado, como por ejemplo, estudios de test-retest para analizar la estabilidad temporal de las puntuaciones. Al respecto, debería reproducirse la administración en situaciones de evaluación lo más idénticas posibles (por ejemplo, en actividades similares en la misma asignatura y clase, o previo a la toma de exámenes de una misma asignatura). Por su parte, y dado que se provee de una medida que valora la motivación situacional hacia actividades académicas, es aconsejable estudiar a futuro los efectos bottom up - top down empleando alguna de las medidas contextuales y globales referidas al área educativa para articular los tres niveles de generalidad. El interés mayor deriva del hecho de que esta temática ha sido ampliamente estudiada en el contexto internacional, hallándose que reiteradas experiencias de motivación autodeterminada hacia actividades concretas pueden impactar sobre un dominio específico -nivel contextualllegando incluso a modificar la orientación general del individuo hacia su entorno -nivel global- y viceversa (Blanchard et al., 2007; Núñez y León, 2018).

Sin perder de vista lo expuesto, se espera poner a disposición de investigadores y profesionales una nueva medida con evidencias de validez y confiabilidad para evaluar adecuadamente la motivación situacional acadé- mica, más congruente con el requisito de especificidad que otras medidas existentes en el contexto internacional. Asimismo, el desarrollo de normas para estudiantes universitarios de Buenos Aires representa una contribución, de cara a la puesta en práctica de estrategias en los ámbitos concretos en que la motivación situacional impacta y tiene lugar: las aulas y su entorno próximo.

\section{Referencias bibliográficas}

Aisenson, G., Valenzuela, V., Celeiro, R., Bailac, K. y Legaspi, L. (2010). El significado del estudio y la motivación escolar de jóvenes que asisten a circuitos educativos diferenciados socioeconómicamente. Anuario de Investigaciones, 16, 109-119.

Baars, G.C.A. y Arnold, I. (2014). Early identification and characterization of students who drop out in the first year at university. Journal of College Student Retention Research Theory and Practice, 16(1), 95-109. doi: 10.2190/ CS.16.1.e

Blanchard, C.M., Mask, L., Vallerand, R.J., de la Sablonnière, R. y Provencher, P. (2007). Reciprocal relationships between contextual and situational motivation in a natural setting. Psychology of Sport and Exercise, 8, 854-873. doi: 10.1016/j.psychsport.2007.03.004.

Boiché, J. y Stephan, Y. (2014). Motivational profiles and achievement: a prospective study testing potential mediators. Motivation and Emotion, 38, 79-92. doi: 10.1007/s11031-0139361-6.

Brenlla, M.E., Messina, V.M. y Aranguren, M. (2013). Adaptación para Buenos Aires de la Escala de Orientaciones Causales (General Causality Orientations Scale - GCOS). Interdisciplinaria, 30(1), 65-84. doi: 10.16888/ interd.2013.30.1.4

Chemolli, E. y Gagné, M. (2014). Evidence against the continuum structure underlying motivation measures derived from Self-Determination Theory. Psychological Assessment, 26(2), 575-585. doi: 10.1037/a0036212.

Deci, E.L. y Ryan, R.M. (1985a). Intrinsic Moti- 
vation and self-determination inhuman behavior. New York: Plenum Press.

Deci, E.L. y Ryan, R.M. (1985b). The General Causality Orientations Scale: Self -Determination in Personality. Journal of Research in Personality, 19, 109-134.

Deci, E.L. y Ryan, R.M. (2000). The "what" and "why" of goal pursuits: Human needs and the self-determination of behavior. Psychological Inquiry, 11, 227-268.

Deci, E.L. y Ryan, R.M. (2004). Handbook of self-determination research. New York: University of Rochester Press.

Elosúa, P. y Zumbo, B.D. (2008). Coeficientes de confiabilidad para escalas de respuesta categórica ordenada. Psicothema, 20(4), 896-901.

Gamboa, V., Valadas, S. y Paixão, O. (2017). Validation of a Portuguese version of the Situational Motivation Scale (SIMS) in academic contexts. Avances en Psicología Latinoamericana, 35(3), 547-557. doi: 10.12804.

Gillet, N., Vallerand, R., Lafrenière, M.A.K. y Bureau, J.S. (2013). The mediating role of positive and negative affect in the situational motivation-performance relationship. Motivation \& Emotion, 37(3), 465-479. doi: 10.1007/ s11031-012-9314-5.

Guay, F., Blais, M.R., Vallerand, R.J. y Pelletier, L.G. (1999). The Global Motivation Scale. Unpublished manuscript, Université du Quebec, Montréal.

Guay, F., Mageau, G.A. y Vallerand, R.J. (2003). On the hierarchical structure of self-determined motivation: A test of top-down, bottom-up, reciprocal, and horizontal effects. Personality and Social Psychology Bulletin, 29, 992-1000. doi: 10.1177/0146167203253297.

Guay, F., Vallerand, R. y Blanchard, C. (2000). On the assessment of situational intrinsic and extrinsicmotivation:The SituationalMotivation Scale (SIMS). Motivation and Emotion, 24(3), 175-213. doi: 10.1023/A:1005614228250.

Horn, J.L. (1965). A rationale and test for the number of factors in factor analysis. Psychometrika, 30(2), 179-185.

Howard, J.L., Gagné, M., Morin, A.J.S. y Forest, J. (2018). Using bifactor explora- tory structural equation modeling to test for a continuum structure of motivation. Journal of Management, 44(7), 2638-2664. doi: $10.1177 / 0149206316645653$.

Lavigne, G.L. y Vallerand, R.J. (2010) The dynamic processes of influence between contextual and situational motivation: A test of the hierarchical model in a science education setting. Journal of Applied Social Psychology, 40, 2343-2359. doi: 10.1111/j.1559-1816.2010.00661.x.

Litalien, D., Morin, A.J.S., Gagné, M., Vallerand, R.J., Losier, G.F. y Ryan, R.M. (2017). Evidence of a continuum structure of academic Self-Determination: A two-study test using a bifactor-ESEM representation of academic motivation. Contemporary Educational Psychology, 51, 67-82. doi: 10.1016/j. cedpsych.2017.06.010.

Lloret-Segura, S., Ferreres-Traver, A., Hernández-Baeza, A. y Tomás-Marco, I. (2014). El análisis factorial exploratorio de los ítems: una guía práctica, revisada y actualizada. Anales de Psicología, 30(3), 1151-1169. doi: 10.5018/ analesps.30.3.199361

Lonsdale, C., Sabiston, C.M., Taylor, I.M. y Ntoumanis, N. (2011). Measuring student motivation for physical education: Examining the psychometric properties of the Perceived Locus of Causality Questionnaire and the Situational Motivation Scale. Psychology of Sport and Exercise, 12, 284-292. doi: 10.1016/j.psychsport.2010.11.003.

López, A. (2008). Regulación motivacional en alumnos de psicología. Un enfoque desde la teoría de la autodeterminación. Tesis de Maestría en Psicología Social. Universidad Nacional de Mar del Plata.

Lorenzo-Seva, U. y Ferrando, P.J. (2013). FACTOR 9.2. A Comprehensive Program for Fitting Exploratory and Semiconfirmatory Factor Analysis and IRT Models. Applied Psychological Measurement, 37(6), 497-498. doi: $10.1177 / 0146621613487794$.

Martin-Albo, J., Núñez, J.L. y Gavarro, J.G. (2009). Validation of the Spanish version of Situational Motivation Scale (EMSI) in the educational context. The Spanish Journal of 
Psychology, 12(2), 799-807. doi: 10.1017/ S113874160000216X.

Ministerio de Educación, Secretaria de Políticas Universitarias. (2013). Anuario 2013. Estadisticas Universitarias. Recuperado de http:// informacionpresupuestaria.siu.edu.ar/DocumentosSPU/Anuario_2013.pdf

Muthén, B. y Kaplan D. (1985). A comparison of some methodologies for the factor analysis of non-normal Likert variables. British Journal of Mathematical and Statistical Psychology, 38, 171-189.

Núñez, J.L. y León, J. (2018). Probando las relaciones entre la motivación global, contextual y situacional: un estudio longitudinal de los efectos horizontal, arriba-abajo y abajo-arriba. Revista de Psicodidáctica, 23(1), 9-16. doi: 10.1016/j.psicod.2017.07.003.

Núñez, J.L., Martín-Albo, J. y Navarro, J. G. (2005). Validación de la versión española de la Échelle de Motivation en Éducation. Psicothema, 17, 344-349.

Ryan, M.R. \& Deci, E.L. (2000). Self-determination theory and the facilitation of intrinsic motivation, social development, and wellbeing. American Psychological, 55(1), 68-78.

Ryan, R.M. y Deci, E.L. (2002). Overview of self-determination theory: An organismic dialectical perspective. En E.L. Deci y R.M. Ryan (Eds.), Handbook of self-determination research (pp. 3-33). Rochester, NY: University of Rochester Press.

Ryan, R.M. y Deci, E.L. (2017). Self-Determi- nation Theory. Basic Psychological Needs in Motivation, Development and Wellness. New York: Guilford Press.

SPSS ICC. (2009). PASW Statistics for Windows, Version 18.0.Chicago: SPSS Inc.

Standage, M., Treasure, D., Duda, J. y Prusak, K. (2003). Validity, reliability, and invariance of the Situational Motivation Scale (SIMS) across diverse physical activity contexts. Journal of Sport \& Exercise Psychology, 25(1), 19-43. doi: 10.1123/jsep.25.1.19.

Stover, J.B., de la Iglesia, G., Rial Boubeta, A. y Fernández Liporace, M. (2012). Academic motivation scale (AMS): Adaptation and psychometric analyses for high school and college students. Psychology Research and Behavior Management, 5, 71-83. doi:10.2147/ PRBM.S33188.

Sturges, D., Maurer, T.W., Allen, D., Gatch, D.B. y Shankar, P. (2016). Academic performance in human anatomy and physiology classes: a 2-yr study of academic motivation and grade expectation. Advances in Physiology Education, 40(1), 26-31. doi: 10.1152/advan.00091.2015.

Vallerand, R.J. (1997). Toward a hierarchical model of intrinsic and extrinsic motivation. Advances in Experimental and Social Psychology, 29, 271-360.

Vallerand, R.J., Blais, M.R., Briere, N.M. y Pelletier, L.G. (1989). Construction et validation de l'Echelle de Motivation en Éducation (EME). Canadian Journal of Behavioural Science, 21, 323-349.

Recibido: 26 de marzo de 2018 Aceptado: 26 de febrero de 2020 